\title{
Videojuegos y educación
}

\author{
Félix Etxeberria Balerdi \\ Universidad del País Vasco \\ hopetbaflsc.ehu.es
}

\section{1.-Breve Historia de los videojuegos [1]}

Los primeros pasos de los actuales videojuegos se detectan en los años 40, cuando los técnicos americanos desarrollaron el primer simulador de vuelo, destinado al entrenamiento de pilotos. En 1962 apareció la tercera generación de ordenadores, reduciendo su tamaño y coste de manera drástica y a partir de ahí el proceso ha sido continuado.

En 1969 nació el microprocesador, que en un reducido espacio producía mayor potencial de información que los grandes ordenadores de los años 50. Es lo que constituye el corazón de nuestros ordenadores, videojuegos y calculadoras.

En 1970 aparece el disco flexible y en 1972 se desarrolla el primer juego, llamado PONG, que consistía en una rudimentaria partida de tenis o ping-pong. En 1977, la firma Atari lanzó al mercado el primer sistema de videojuegos en cartucho, que alcanzó un gran éxito en Estados Unidos y provocó, al mismo tiempo, una primera preocupación sobre los posibles efectos de los videojuegos en la conducta de los niños.

Tras una rápida evolución, en la que el constante aumento de la potencia de los microprocesadores y de la memoria permitieron nuevas mejoras, en 1986, la casa Nintendo lanzó su primer sistema de videojuegos que permitió la presentación de unos juegos impensables nueve años atrás. La calidad del movimiento, el color y el sonido, asî como la imaginación de los creadores de juegos fueron tales que, unidos al considerable abaratamiento relativo de dichos $\mathrm{VJ}$, a comienzos de los 90 , en nuestro país se extendieron de manera masiva los juegos creados por las dos principales compañías, Sega y Nintendo, pasando en poco tiempo a constituirse en uno de los juguetes preferidos de los niños. [2]

La extensión masiva de los $\mathrm{VJ}$ en los años 90 ha provocado una segunda oleada de investigaciones, desde la medicina, la sociología, la psicología y la educación, además de la preocupación y las valoraciones que dichos juegos han recibido por parte de padres, educadores y principalmente los medios de comunicación, para quienes generalmente los VJ son vistos como algo negativo y perjudicial. Las más prestigiosas universidades, revistas y publicaciones están haciendo un hueco a la preocupación por uno de los temas preferidos a la hora de elegir los juegos, no solo de los niños y adolescentes, sino también de jóvenes y adultos.

\section{2.-Tipos de videojuegos}

Si bien el mercado de los VJ es algo en constante cambio, podemos decir que existen una serie de juegos que tienen características comunes y que permiten clasificar a los mismos en torno a las siguientes categorías (Estallo, 1995): 


\begin{tabular}{|c|c|c|}
\hline $\begin{array}{l}\text { TIPO DE } \\
\text { JUEGO }\end{array}$ & CARÁCTERISTICAS & MODALIDADES \\
\hline ARCADE & $\begin{array}{l}\text { Ritmo rápido de juego } \\
\text { Tiempo de reacción mínimo } \\
\text { Atención focalizada } \\
\text { Componente estratégico } \\
\text { secundario }\end{array}$ & \begin{tabular}{||l} 
Plataformas \\
Laberintos \\
Deportivos \\
Dispara y olvida
\end{tabular} \\
\hline SIMULADORES & $\begin{array}{l}\text { Baja influencia del tiempo de } \\
\text { reacción } \\
\text { Estrategias complejas y } \\
\text { cambiantes } \\
\text { Conocimientos específicos } \\
\end{array}$ & \begin{tabular}{||l} 
Instrumentales \\
Situacionales \\
Deportivos
\end{tabular} \\
\hline ESTRATEGIA & $\begin{array}{l}\text { Se adopta una identidad } \\
\text { específica } \\
\text { Solo se conoce el objetivo final } \\
\text { del juego } \\
\text { Desarrollo mediante ordenes y } \\
\text { objetos } \\
\end{array}$ & \begin{tabular}{|l} 
Aventuras gráficas \\
Juegos de rol \\
Juegos de guerra
\end{tabular} \\
\hline \begin{tabular}{|l} 
JUEGOS DE \\
MESA
\end{tabular} & $\begin{array}{l}\text { Cartas, ajedrez, etc.Pin pon, } \\
\text { petacos, etc }\end{array}$ & Trivial Pursuit \\
\hline \begin{tabular}{|l} 
OTROS \\
JUEGOS
\end{tabular} & & \\
\hline
\end{tabular}

Hay que tener en cuenta que la clasificación que hacen las revistas especializadas difiere en cierto sentido de estas grandes categorías, variando además con bastante frecuencia. En la actualidad (Hobby Consolas, 1998), los especialistas en el tema distribuyen a los juegos con arreglo a varios parámetros, como el tipo de consola, nivel de dificultad, tipo de juego, etc. Según esto contamos con 5 tipos de consolas en el mercado español: Game Boy, Nintendo 64, PSX Platinun, Sega Return y PlayStation. A estos tipos de consolas hay que añadirles los juegos que tienen como soporte un ordenador personal.

\section{3.-Características de los jugadores de VJ}

¿Qué tipo de juegos son los más utilizados por los jugadores? ¿Cuánto tiempo dedican al juego? ¿Dónde y cómo practican el juego?

Al utilizar el término jugador es difícil reducir esa expresión a unos determinados sujetos que juegan de manera uniforme. Los usuarios tienen sus preferencias, sus peculiaridades y sus costumbres. Pasemos a ver algunas de estas cualidades diferenciadoras en lo relativo a los jugadores

\section{Los videojuegos: uno de los juguetes más elegidos}

Ya hemos dicho más arriba que, aunque llevan poco tiempo desde que se introdujeron en España, ya en el año 1992, los videojuegos ocupaban uno de los primeros lugares en cuanto a la elección de los niños, llegando a superar el $40 \%$ de los juguetes que se vendieron, con unas ventas que rondaban los 50.000 millones de pesetas. Según el estudio realizado por la Universidad de Valencia (1992) el videojuego ocupaba el primer lugar entre los juguetes más preferidos para el $62 \%$ de los niños españoles, situándose en 
segundo lugar en el caso de las niñas, por detrás de los juegos de misterio. Si bien esta tendencia al crecimiento parece haberse estancado, los VJ siguen siendo uno de los objetos más preferidos por los niños y adolescentes.

\section{Los videojuegos preferidos}

No todos los videojuegos reciben la misma aceptación por parte de los usuarios. Las preferencias de éstos se dividen según el tema del juego y la estructura formal del mismo. Atendiendo a la clasificación temática realizada por Funk (1993), los juegos preferidos por los adolescentes fueron los siguientes:

$\begin{array}{lc}\begin{array}{l}\text { De violencia } \\ \text { fantástica }\end{array} & 32 \% \\ \text { Deportivos } & 29 \% \\ \text { Temas generales } & 20 \% \\ \begin{array}{l}\text { De violencia } \\ \text { humana }\end{array} & 17 \% \\ \text { Educativos } & 2 \%\end{array}$

Vemos, como primera nota destacable, que los juegos catalogados como "educativos" reciben una muy baja valoración por parte de los niños y adolescentes, mientras que quienes tienen como tema la violencia ocupan una primera situación con cerca de un 50 $\%$ del mercado;

Tras el análisis de los principales juegos del mercado, realizado por la revista especializada Hobby Consolas $\left(\mathrm{n}^{\circ} 23,1998\right)$, se puede comprobar que el $57 \%$ de los 272 juegos examinados tiene un carácter violento, con luchas, peleas o guerras. El otro $43 \%$ lo componen los juegos de carreras de coches y motos, el futbol y basket y finalmente otro tipo de juegos de diversión neutra.

Teniendo en cuenta la estructura formal de los VJ, arcades, simulación, aventuras y juegos de mesa, la distribución de las preferencias de los adolescentes (Estallo, 1995) es la siguiente:

$\begin{array}{ll}\text { Arcade } & 42 \% \\ \text { Simuladores } & 25 \% \\ \text { Aventuras } & 28 \% \\ \begin{array}{ll}\text { Juegos de } \\ \text { mesa }\end{array} & 5 \% \\ & \end{array}$

Es de destacar también el escaso lugar que ocupan los juegos de mesa (parchis, ajedrez, etc.) en la elección de los usuarios de los VJ.

De todas maneras, las preferencias de los usuarios de los videojuegos varían constantemente en razón de los tipos de juegos, las modas, las campañas y otra serie de variables muy complejas, de tal modo que es difícil establecer unas prioridades en la elección de los juegos con validez permanente. Algunas revistas especializadas (Hobby Consolas) publican todos los meses la lista de novedades en España, en las que incorporan más de 20 nuevos juegos, y también facilitan el catálogo de éxitos de los juegos en el mercado, indicando cuáles son los más vendidos en cada uno de los formatos o tipos de consolas. Como indicador de las aficiones de los usuarios y del carácter que 
tienen los juegos más elegidos, mostramos a continuación los elegidos como mejores juegos del año 1997 (Hobby Consolas, 1998):

\begin{tabular}{|l||c|}
\hline Mejores juegos 1997 & Características \\
\hline Final Fantasy VII & Peleas \\
\hline \hline Super Mario 64 & Peleas \\
\hline Tomb Raider 2 & Peleas \\
\hline FIFA & Fútbol. \\
\hline \hline Goldeneye & Peleas \\
\hline $\begin{array}{l}\text { Dragon Ball Final } \\
\text { About }\end{array}$ & Peleas \\
\hline
\end{tabular}

Fuente: Hobby Consolas, 1998.

Es necesario resaltar el hecho de que entre los seis juegos más votados por los usuarios de los videojuegos, cinco resultan relacionados con luchas, violencia y peleas. El único juego que no es de peleas y lucha, en cuarto lugar, está dedicado al deporte del fútbol.

En cuanto a las preferencias de los chicos y chicas respecto a los videojuegos, según Gros (1998), los juegos preferidos por los niños y niñas de 7 centros de enseñanza primaria eran los siguientes:

\begin{tabular}{||c|c||}
\hline Niños & Niñas \\
\hline \hline 1.-PC Fútbol & 1.-Super Mario \\
\hline \hline $\begin{array}{c}\text { 2.-Street } \\
\text { Figther }\end{array}$ & 2.-Sonic \\
\hline $\begin{array}{c}\text { 3.-Bola de } \\
\text { Dragón }\end{array}$ & 3.-Tetris \\
\hline \hline 4.-Super Mario & 4.-Street Figther \\
\hline \hline $\begin{array}{c}\text { 5.-Mortal } \\
\text { Kombat }\end{array}$ & 5.-El Rey León \\
\hline \hline
\end{tabular}

Comprobamos en las preferencia de los chicos y chicas que se observa una mayor inclinación de los chicos por los juegos deportivos y violentos, mientras que las chicas se decantan más por juegos en los que la violencia está mucho menos presente.

\section{Cuánto tiempo juegan los usuarios}

Un tema de constante estudio es el de la medición del número de horas que los jugadores le dedican a los VJ y las posibles repercusiones que para la vida diaria y los estudios puedan tener dichas horas. Esta cuestión es relativamente fácil de resolver, puesto que son constantes las encuestas al respecto y la contabilización de las mismas no deja lugar a dudas de interpretación. 
Los constantes sondeos que se realizan indican una tendencia creciente en el número de horas que se dedican a los VJ y a la televisión en general. De año en año, el tiempo que los niños y adolescentes dedican a la pantalla sigue en aumento.

En el estudio realizado por Funk (1993) se nos ofrece una clasificación de los usuarios en base al número de horas semanales y en razón del sexo del jugador, en la sociedad americana.

\begin{tabular}{|c||c|c|c|c||}
\hline Horas/Semana & 1 - 2 horas & 3 - 6 horas & $\begin{array}{c}\text { Más de 6 } \\
\text { horas }\end{array}$ & Nada \\
\hline \hline Chicos & $36 \%$ & $29 \%$ & $23 \%$ & $12 \%$ \\
\hline \hline Chicas & $42 \%$ & $15 \%$ & $6 \%$ & $37 \%$ \\
\hline
\end{tabular}

Fuente (Funk, 1993)

En otro estudio realizado por Estallo (1995) se analizó una muestra de 278 sujetos entre 12 y 33 años, seleccionados al azar entre escolares y adultos en España. El número de mujeres era de 146 y el de varones de 132 .

Clasificando a los sujetos en tres categorías, según su mayor o menor dedicación al juego, la distribución de la muestra fue la siguiente:
Anecdóticos (1 vez al mes o menos)
Regulares (entre 3 veces al mes y 1 a la semana) $23 \%$
Habituales (entre más de 1 vez a la semana y más de 1 vez al día)

El reparto según el sexo se hace de la siguiente manera:

\begin{tabular}{||l||c|c|c||}
\hline & $\begin{array}{c}\text { Anecdóticos } \\
\mathbf{5 8 \%}\end{array}$ & $\begin{array}{c}\text { Regulares } \\
\mathbf{2 3 \%}\end{array}$ & $\begin{array}{c}\text { Habituales } \\
\mathbf{1 9 \%}\end{array}$ \\
\hline \hline Chicos & 36 & 37 & 27 \\
\hline \hline Chicas & 78 & 22 & 12 \\
\hline
\end{tabular}

Fuente (Estallo, 1995)

La frecuencia de juego en relación al sexo nos indica que los varones dedican más tiempo a los VJ y que su frecuencia de juego es mayor que en las mujeres. En casi todos los estudios que se realizan sobre este tema hay coincidencia al asegurar que al hablar de diferencias en cuanto al sexo, los jugadores sobrepasan a las jugadoras en el número de horas que dedican al juego.

En este punto, uno de los problemas que suele plantearse respecto al uso de los VJ es el exceso de tiempo dedicado al mismo, la adicción o lo que en otros términos viene a llamarse ludopatía, o patología relacionada con una afición incontrolada por el juego. Pues bien, los usuarios a los VJ desarrollan también una ludopatía específica que, según la Asociación Navarra de Ludópatas, afecta a un $5 \%$ de los niños. Según dicha asociación, estos adictos a los videojuegos corren un riesgo muy grande de que pasen a ser adultos ludópatas. 
Por grupos de edad, la distribución de la muestra con respecto a la frecuencia de juego nos indica que los más jóvenes son quienes tienen una mayor inclinación a los videojuegos, mientras que los más mayores ven disminuir su afición.

\begin{tabular}{|l|c|c||c||}
\hline & $\begin{array}{c}\text { Anecdóticos } \\
\mathbf{5 8 \%}\end{array}$ & $\begin{array}{c}\text { Regulares } \\
\mathbf{2 3 \%}\end{array}$ & $\begin{array}{c}\text { Habituales } \\
\mathbf{1 9 \%}\end{array}$ \\
\hline \hline Adolescentes & 33 & 29 & 48 \\
\hline Jóvenes & 29 & 20 & 23 \\
\hline \hline Adultos & 48 & 11 & 4 \\
\hline
\end{tabular}

Fuente (Estallo, 1995)

Es evidente que los jugadores son más habituales y le dedican más tiempo al VJ a medida que decrece su edad, siendo los jugadores de más edad quienes menos horas practican el juego.

Es importante anotar que, a estos datos, debemos añadir el número de horas diarias que dedican a ver la televisión los niños y adolescentes. Casi todos los estudios coinciden al afirmar que la media está comprendida entre 3 y 4 horas diarias.

Estos datos nos indican que el conjunto de los videojuegos y la televisión ocupan más tiempo que el que se dedica a la escuela, el trayecto al colegio y la realización de las tareas escolares, tal y como se viene confirmando a lo largo de casi todos los estudios que se realizan sobre el tiempo empleado por los niños y adolescentes.

\section{Jugar solo o acompañado}

Una cuestión que tiene bastante importancia a la hora de analizar la modalidad de juego de los usuarios es la de comprobar la manera en que éstos juegan, desde el punto de vista social; es decir, si juegan solos o acompañados. Puesto que muchas de las críticas que se realizan al uso de los videojuegos se centran en que fomentan el individualismo y obstaculizan la sociabilidad, será procedente analizar de qué manera se juega. En el estudio realizado por Estallo (1995), la clasificación de la modalidad de juego, atendiendo a la edad del jugador y al sexo del mismo, es la siguiente:

\begin{tabular}{|l||c||c||}
\hline & $\begin{array}{c}\text { Juegan solos } \\
\mathbf{3 0 \%}\end{array}$ & $\begin{array}{c}\text { Juegan } \\
\text { acompañados } \\
\mathbf{7 0 \%}\end{array}$ \\
\hline \hline Adolescentes & 35 & 65 \\
\hline Jóvenes & 30 & 70 \\
\hline \hline Adultos & 26 & 74 \\
\hline
\end{tabular}

\begin{tabular}{||l||c||}
\hline Juegan solos & $\begin{array}{c}\text { Juegan } \\
\text { acompañados }\end{array}$ \\
\hline
\end{tabular}




\begin{tabular}{||l||c||c||} 
& $\mathbf{3 0 \%}$ & $\mathbf{7 0 \%}$ \\
\hline Chicos & 34 & 66 \\
\hline Chicas & 27 & 73 \\
\hline
\end{tabular}

Fuente (Estallo, 1995)

Una primera conclusión que podemos sacar a la luz de estos datos es que, la mayoría de los jugadores lo hacen acompañados, el $70 \%$, mientras que un $30 \%$ juega de modo individual. Cuanto más edad tiene el usuario más tendencia tiene a jugar en compañía, al tiempo que las chicas juegan más acompañadas que los chicos.

Hay que destacar, por otra parte, que la mayoría de los juegos contemplan la posibilidad de jugar uno o más jugadores.

\section{4.-Las razones del éxito de los videojuegos.}

El éxito extraordinario de los videojuegos y su progresiva implantación entre los niños, jóvenes y adultos merece una atención especial, puesto que algo debe haber en este tipo de actividad que atraiga y mantenga de una manera tan intensa el interés de los usuarios.

Dos son las razones, a nuestro juicio, del gran eco que los videojuegos tienen en nuestra sociedad. Por una parte, hay que tener en cuenta la gran afinidad que existe entre los valores, actitudes y comportamientos que promueven los videojuegos y los que son imperantes en nuestra sociedad actual. Por otra parte, desde el punto de vista del aprendizaje, hay que tener en cuenta que los videojuegos cumplen muchos de los requisitos que una eficaz enseñanza debe contemplar, y en muchos casos lo hacen mejor incluso que nuestros actuales sistemas educativos.

\section{1.-Afinidad con los valores dominantes.}

Muchos de los valores dominantes en nuestra sociedad se encuentran presentes en los videojuegos y programas de televisión en general. Hablamos del sexismo, la competición, el consumismo, la velocidad, la violencia, la agresividad, etc. Hay una gran sintonía entre los valores promovidos por estos juegos y los que están presentes en nuestro entorno social, de manera que los comportamientos que se practican en estos juegos son los que encuentran un mayor apoyo y aceptación social. Puede decirse también, a la inversa, que nuestros niños y jóvenes van aprendiendo y socializándose en estos valores y actitudes a través de los videojuegos y los programas de televisión.

Dentro de los valores y actitudes más impulsados por los videojuegos, destacamos algunos de ellos por su especial interés:

a).-La competitividad. Es uno de los ejes de nuestra sociedad, presente en todos los niveles y todos los ámbitos, en la empresa, el deporte, la familia, etc. Ocupa un papel importantísimo en la infraestructura de los videojuegos, tanto en la competición con otros como en la competición con uno mismo.

b).-La violencia es otra de las dimensiones que tienen un gran hueco en el conjunto de los videojuegos y que, lamentablemente, está también muy presente en nuestra sociedad, puesto que vivimos un entorno violento, sobre todo a través de la televisión, en donde se destaca como tema estelar en las películas, telediarios, etc. 
c).-Sexismo y erotismo. La utilización del sexo para conseguir objetivos comerciales y la difusión y promoción de los roles sexuales diferenciados y en relación de dependencia, tiene también un fuerte eco en los juegos de pantalla, al igual que son utilizados por la publicidad diaria con el fin de conseguir objetivos económicos.

d).-Velocidad. Es otra de las características de nuestra sociedad moderna, que fomenta el impulso de correr más rápido que nadie, al tiempo que parece impotente para reducir las muertes por accidentes de circulación. Numerosos juegos muestran este aspecto competitivo relacionado con la velocidad de coches, motos y otros vehículos, en total consonancia con lo que ocurre en la vida real.

e).-Consumismo. La iniciación en el mundo de los videojuegos supone un fuerte impulso para el desarrollo de actitudes y comportamientos consumistas, con la compra de aparatos, accesorios, cambios de modas, revistas especializadas, infraestructuras, ordenadores, etc.

\section{2.- Psicología del aprendizaje y Videojuegos.}

Desde el punto de vista psicoeducativo, es interesante analizar el éxito de los VJ a la luz de las teorías de la motivación y del aprendizaje. Vamos a analizar el paralelismo entre el aprendizaje social y el sistema de motivación y aprendizaje implícito en los videojuegos. ¿Cuál es la clave del éxito de los videojuegos? ¿Qué condiciones se dan en ellos que no se cumplen en la familia o en la escuela?.

Según Bandura (1984) y Castillejo (1987), hasta hace poco algunos teóricos mantenían que los determinantes principales de la conducta son las fuerzas motivacionales que se configuran en necesidades, tendencias e impulsos que, con frecuencia, operan por debajo del umbral de la conciencia. En resumen, se trataría de fuerzas interiores. Al desarrollar la teoría de la conducta, el análisis causal cambió de perspectiva: se paso de propugnar unos determinantes internos amorfos a analizar en detalle cuáles son las influencias externas de las respuestas humanas; es decir, se cambia el punto de vista, inclinándose hacia las fuerzas del medio. La polémica parecía centrada en la prioridad por las fuerzas internas o por el medio; en la herencia y en el ambiente.

Pero en los últimos años, la comprensión de los procesos psicológicos ha progresado sustancialmente y se hace necesario reformular algunas suposiciones acerca de la adquisición de la conducta humana. Es en esta línea en la que la "Teoría del aprendizaje social" aporta una serie de adquisiciones que permiten explicar mejor la conducta, en el sentido de que se reconoce que el sujeto no se limita a reaccionar ante el entorno, y que juegan un papel importante la observación, la capacidad humana de emplear símbolos y procesos cognitivos y la capacidad de autorregulación de los sujetos. De modo sintético, la contribución de la Psicología del Aprendizaje Social al proceso de enseñanzaaprendizaje es la siguiente:

1.-Se reconoce que la observación puede influir notablemente en los pensamientos, los afectos y las conductas de los hombres. Se acentúa la importancia de los procesos vicarios, simbólicos y auto-regulatorios en el funcionamiento psicológico.

2.-La capacidad humana de emplear símbolos permite representar los fenómenos, analizar su experiencia consciente, planear, imaginar, y actuar de manera previsora.

3.-Los procesos de autorregulación juegan un papel central, seleccionando, organizando y filtrando las influencias externas. El sujeto no se limita a reaccionar. 
4.-Hay una interacción continua entre el sujeto y el entorno, haciendo que la persona influya en su destino y que se establezcan los límites de esa autonomía.

\section{Las fuentes de la motivación y de la conducta}

Para la teoría del aprendizaje social, las fuentes de la motivación y de la conducta en las personas se centran en torno a estos factores:

a).- Los modelos. Sería muy laborioso y peligroso aprender todo por las propias acciones. La mayor parte de las conductas las aprendemos a través de la observación, por medio del modelado, la imitación o el aprendizaje vicario. De este modo evitamos errores, aprendiendo con ejemplos. Refranes como "donde fueres haz lo que vieres" y otros más nos indican el valor de este tipo de aprendizaje. Los modelos pueden ser más o menos participativos. El aprendizaje vicario, aprender viendo hacer a otros, se revela como un sistema eficaz de aprendizaje. Un modo de aprender la forma de servirse en un comedor público es observar cómo resuelven el problema las personas que nos preceden.

b).-Los reforzadores. Un reforzador es todo aquello que aplicado a una determinada conducta incrementa o disminuye la probabilidad de su aparición. Los tipos de reforzadores son variados:

- Las consecuencias de las respuestas. El aprendizaje más rudimentario está basado en la experiencia directa y es el que se debe a los efectos positivos y negativos que producen las acciones. Cuando las personas se enfrentan a los sucesos cotidianos, algunas de sus respuestas tienen éxito, mientras que otras no tienen ningún efecto o tienen como resultado el castigo. A través de este proceso de reforzamiento diferencial, llega finalmente un momento en el que se seleccionan las formas de respuesta que han tenido éxito y se descartan las que han sido ineficaces. Se afianzan las respuestas que provocan placer o satisfacción, mientras que se rehuyen las conductas que son fuente de dolor o de insatisfacción. Algunos sujetos se ejercitan tocando el piano por el placer que obtienen de su práctica.

- Los refuerzos extrínsecos, son consecuencias de la conducta que tienen una relación arbitraria con la misma, como por ejemplo el dinero, ventajas y privilegios, los castigos, la aprobación, etc. Los refuerzos extrínsecos aparecen también como una gran fuente de motivación en la conducta y la vida cotidiana está también plagada de este tipo de refuerzos. En algunos casos, al margen de la satisfacción personal que obtiene, el pianista se esfuerza en su actividad por conseguir un premio o el aplauso del público.

- También son refuerzos extrínsecos los llamados "amplificadores sociales", es decir, los que aumentan los efectos, positivos o negativos, de la conducta del sujeto, en el contexto, en los demás y en él mismo. (Castillejo, 1987).

- El control cognitivo es un tipo de refuerzo que tiene una influencia también considerable. Cuando los sujetos conocen las tareas a realizar, conocen la meta o el objetivo a conseguir, conocen los refuerzos existentes, su cadencia o sistema, los resultados de sus acciones y el nivel que están consiguiendo tienden a reforzar y consolidar una determinada conducta.

\section{c).- La práctica}

La realización de tareas concretas ayuda a la consecución de mejores respuestas por parte 
de los sujetos implicados. Las tareas pueden ser directas (solucionar problemas, construir, etc) o bien simbólicas (imaginar, prevenir soluciones, prevenir, hacer una simulación, etc). Cuanto más prácticas sean las tareas y más sentidos se impliquen en ellas, vista, oído, manipulación, etc. la actividad realizada tiene una mayor capacidad de afianzarse.

\section{d).- El clima}

Al referirnos al clima hablamos del modo en que el medio provoca estimulaciones que obligan al sujeto a adoptar un tipo de conducta. Si el medio facilita la conducta deseada, la probabilidad de aparición de ésta es más alta. Tanto el aspecto contextual, relativo a los materiales, condiciones físicas, luz, temperatura, ambiente ecológico, etc, así como el aspecto psicosocial, es decir el grado de autonomía, la actividad en grupo, el ambiente de cooperación o competición, etc. tienen una influencia muy grande en el comportamiento de los sujetos.

\section{En resumen}

El funcionamiento de la motivación y su influencia en el aprendizaje puede ser condensado en las siguientes reglas:

1. Lo fundamental es que la tarea tenga en sí misma el suficiente atractivo o motivación para promover el aprendizaje.

2. En caso contrario, existen otras fuentes de motivación, entre las que destacan los refuerzos, que pueden ejercer un papel importante. Estos refuerzos pueden tener características de tipo material (premios, recompensas, dinero, etc.), psicológico (alabanzas), intelectual (conocer las tareas y los resultados), y social (reconocimiento social, amplificadores sociales)

3. El sistema de refuerzos tiene una mayor influencia cuando se cumplen determinados requisitos:

- Que tengan carácter positivo, recompensador, en lugar de castigo.

- Que sea un programa definido, no arbitrario, de refuerzo.

- Que suponga una dificultad progresiva

- Que el refuerzo o la recompensa sea inmediata

- Que esté adaptado a las características y ritmo del individuo (niveles)

- Que se conozcan los resultados rápidamente

- Que tengan un reconocimiento social.

Si comparamos la realidad de los VJ y el análisis de la motivación que se desarrolla en la "Teoría del Aprendizaje Social", podemos comprobar que los juegos electrónicos reúnen muchas de las características que exige una organización eficaz del aprendizaje social. En el capítulo de los reforzadores, parece que los videojuegos contienen dentro de su esquema una gran cantidad de reforzadores:

- El carácter lúdico de los aprendizajes

- La dificultad creciente y progresiva de las habilidades 
- El ritmo individual de cada participante

- El conocimiento inmediato de los resultados

- El conocimiento claro de las tareas y objetivos a conseguir

- La posibilidad de repetir y corregir el ejercicio

- La recompensa inmediata después de cada logro

- El conocimiento de que existe un sistema determinado y definido de recompensas

- El reconocimiento social de los logros adquiridos (compañeros, boleras, etc.)

- La posibilidad de inscribir los records o niveles máximos

- La constante superación del propio nivel

- Los aplausos, los gritos del publico

- La gratificación del beso de la chica, etc

- La actividad participativa, manual, cardiovascular, etc.

- La identificación con héroes socialmente prestigiosos, Rambo, etc.

- La práctica de deportes o actividades socialmente valoradas, futbol, automovilismo

- La estimulación visual, auditiva, kinestésica, actitudinal, etc. de los juegos.

Al contrastar esta actividad de los VJ con otras actividades realizadas en el aula o en el hogar, comprobamos que el desequilibrio en cuanto a la utilización de los recursos para la motivación de la conducta es totalmente favorable hacia los VJ. Ni en la escuela ni en la familia se realizan, por lo general, actividades lúdicas que por sí solas produzcan gran satisfacción, ni existe un conocimiento exacto de los fines a conseguir, ni un refuerzo inmediato y constante por los logros conseguidos, ni una actividad programada para desarrollarse con una dificultad progresiva. Tanto en la escuela como en la familia se realizan muchas veces tareas rutinarias, para las que no existen estímulos tan intensos como en el VJ (luces, sonidos, manipulación), que casi nunca reciben una recompensa, mientras que por el contrario son más abundantes los silencios o los castigos. La maquina, el videojuego, nos hace una demostración de cómo se juega, nos plantea claramente las reglas del juego, nos permite jugar al nivel adecuado a nuestras posibilidades, nos facilita el progresar continuamente, nos invita a manipular y a manejar instrumentos y resolver problemas, nos dice inmediatamente el nivel que hemos conseguido, nos da recompensas si cumplimos determinados requisitos, nos dice cuándo hemos alcanzado el record, nos permite inscribirlo públicamente, nos aplaude, nos anima, ... en suma, nos da la oportunidad de sentirnos héroes, en algunos casos de identificarnos con héroes reconocidos socialmente, como Rambo, de ser un poco "mejor" cada día. Esta serie de circunstancias sólo se dan en los videojuegos y no se dan en la vida escolar ni en la familiar de una manera tan intensa.

La competición es una fuente intensa de motivación, tal y como queda evidenciado en la vida diaria con los deportes, olimpiadas, concursos y la vida laboral. Los videojuegos están basados en una fuerte dosis de competición que se refleja en varios niveles, que pueden manifestarse simultáneamente. Existe competición entre el jugador y la maquina, como en el ajedrez. También se puede dar entre el jugador y quienes han inscrito su 
record anterior en el juego, entre el jugador y sus compañeros presentes, entre el jugador y su propio record (autoemulación). En fin, el VJ permite al niño competir con su padre o con su madre en algo que, quizás, va a ser la primera actividad en la que va a poder superarle. Probablemente, un niño o niña de 10 años, supera a sus padres en el dominio y las puntuaciones de los videojuegos, y es seguramente una de las primeras y pocas actividades en las que puede manifestarse esa superioridad ante sus padres. Cuando esto ocurre, la satisfacción de los niños es de una magnitud enorme.

La constante repetición es una de las formas de afianzar comportamientos y de permitir mayor dominio de la actividad cada vez. Por ello, a fuerza de repetición y de jugar durante muchas horas, los niños y adolescentes llegan a convertirse en grandes expertos y alcanzan progresivamente puntuaciones o niveles superiores. El videojuego les muestra diariamente, de modo palpable y cuantificable, todo lo que están progresando, de manera que cada día que avanza consiguen un mejor nivel.

\section{5.-Algunos tópicos sobre la influencia negativa de los VJ}

Está muy extendida la creencia de que los videojuegos tienen una incidencia negativa sobre muchos aspectos de la personalidad de los jugadores, y especialmente sobre los niños. Es muy frecuente observar en revistas, periódicos y otros medios de comunicación afirmaciones contundentes respecto, generalmente, a las nefastas consecuencias que el uso de los VJ tiene sobre la inteligencia, la personalidad o la dimensión social de los jugadores.

El origen de esta corriente de opinión se inicia con el creciente aumento de la afición de los niños por los VJ y la incorporación progresiva de características violentas o agresivas en los nuevos juegos. Desde que en el año 1973 se creó el juego de Pong, simulando una rudimentaria partida de tenis, y después la creación de los Space Invaders (matamarcianos), Pac-Man (come-cocos), y otros similares, se ha comenzado una "carrera armamentística" que ha dado paso a juegos en los que el componente violento, bélico y agresivo ha ido adquiriendo cada vez más intensidad y más realismo. Los juguetes de guerra actuales son "casi reales", como el de la Guerra del Golfo; las peleas callejeras son casi humanas, en cuanto a sus movimientos y la sangre que se esparce por la pantalla; los combates entre humanos o entre seres de otros mundos son cada vez más brutales, tal como "Mortal Kombat", en el que la versión II supera en crueldad a la versión I. La versión actual de Mortal Kombat es la IV (1998).

Existe la opinión generalizada de que este tipo de juegos no puede aportar nada bueno en el desarrollo psicosocial, sobre todo en el caso de los más niños. Pero la verdad es que, muchas veces, las opiniones y los juicios vertidos se han hecho en base a consideraciones intuitivas y no en relación a investigaciones serias [3]

A pesar de la poca consistencia "científica" de estas afirmaciones, ¿Es necesario demostrar de modo riguroso que el juego frecuente con este tipo de entretenimientos puede llevar emparejado algún tipo de riesgo?. Muchos padres y educadores no necesitan grandes demostraciones para saber que la convivencia con la violencia no aporta beneficios al desarrollo infantil.

Del conjunto de las preocupaciones que el uso, y sobre todo el abuso, de los VJ provoca en los educadores destacamos los siguientes problemas:

\section{La violencia}

La mayoría de los autores y de los investigadores, así como padres y resto de personas 
opinan que los videojuegos están muy cargados de violencia y agresividad y que repercuten negativamente sobre el comportamiento de los niños y adolescentes.

En algunos casos se levantan voces sobre el papel catártico de los videojuegos violentos, añadiendo que en lugar de fomentar la violencia lo que hacen es encauzarla y darle salida.

No falta también quien piensa que la violencia de los nuevos juegos no es superior a la que se ha representado desde hace mucho tiempo a través de la literatura infantil universal, el cine, los comics, etc. El cuento del lobo, blancanieves, pulgarcito, y otra serie de clásicos vendrían a ser el antecedente de los actuales modos de jugar con los ordenadores.

\section{El sexismo}

Se afirma que la mayoría de los videojuegos representan a personajes masculinos, que los chicos son los principales usuarios de estos juegos, que las pocas figuras femeninas que aparecen lo hacen en una situación de inferioridad, de segundo plano, de cautivas que hay que rescatar, en actitudes de sumisión, mientras que los personajes masculinos están representados de forma activa, valiente y dominadora.

\section{El racismo}

Se acusa a los videojuegos de fomentar los estereotipos raciales, situando en posición de inferioridad o de mayor peligrosidad a los pocos personajes que no sean blancos.

\section{La sociabilidad}

Hay una tendencia a considerar que los usuarios de los videojuegos son personas poco sociales, que tienen dificultades de relación con gente de su edad y que se refugian en los juegos por esas dificultades.

\section{La creatividad}

También se afirma que el juego en las consolas o los ordenadores promueve la actividad repetitiva y poco imaginativa.

\section{Los trastornos del carácter}

En algunos casos, se llega a defender que la práctica de los videojuegos, en algunos casos llevada a su extremo, puede provocar grandes trastornos de la personalidad, como psicosis (o la hiperestesia, en el caso extensamente difundido de un niño italiano)

\section{La inteligencia}

En otros casos, se afirma que la inteligencia se embota, que la mente del niño queda bloqueada y que apenas puede desarrollar sus actividades con normalidad.

\section{6.-La investigación y los videojuegos.}

¿Qué hay de cierto en todo lo que hemos visto anteriormente? ¿Son los videojuegos sexistas, violentos y asociales?. 
Los primeros videojuegos fueron introducidos en los años 70 y hacia el final de esa década se convirtieron ya en uno de los juegos preferidos por los niños. La respuesta mayoritaria de los padres fue de preocupación ante los posibles efectos perniciosos de los videojuegos sobre los niños. Pero las primeras investigaciones sobre el tema no fueron concluyentes.

Sin embargo, con el resurgimiento de los videojuegos en los años 80, a raíz de la introducción del sistema Nintendo, el interés por los efectos de los VJ se ha visto reforzado y con él la necesidad de realizar nuevas investigaciones. Algunas de estas investigaciones sugieren que el jugar con los VJ puede afectar físicamente a los niños, desde provocar epilepsia, cambios de la presión sanguínea, el ritmo cardiaco, etc. Sin embargo, los trastornos serios de tipo fisiológico se limitan a un número reducido de jugadores.

Otras investigaciones han encontrado efectos beneficiosos de los VJ, desde el desarrollo de la creatividad y actitudes prosociales hasta la rehabilitación física y oncológica (Funk, J.B. 1993)

Hay autores como Provenzo (1991) que defiende la tesis de que la investigación realizada sobre los VJ hasta la fecha es deficiente y que pone en duda los supuestos efectos, generalmente negativos, de la práctica de dichos juegos. Afirma que muchas investigaciones están inconclusas o diseñadas inadecuadamente a largo término. Propone un análisis más serio del problema y sobre todo el estudio de las posibilidades de la utilización de los VJ en la enseñanza.

Estallo, (1995) después de realizar una valoración de las afirmaciones más o menos gratuitas y las investigaciones más rigurosas, llega a la conclusión de que la mayoría de las sentencias reflejadas en periódicos y revistas no tienen ningún fundamento científico. Considera que las investigaciones no avalan ninguno de estos males catastróficos que los críticos aficionados denuncian. Así pues, según este psicólogo, no hay evidencia de problemas intelectuales, violencia, sexismo, etc. que pueda ser justificada desde el punto de vista científico.

Llegados a este punto, ante la falta de acuerdo sobre los efectos nocivos o beneficiosos de la práctica de los VJ, hemos creído conveniente plantearnos el realizar una revisión de las investigaciones existentes sobre el tema, con el fin de ver si es que hay evidencias en uno u otro sentido. En nuestra opinión, si bien es cierto que se ha exagerado, al afirmar de manera "intuitiva" los males de la utilización de los $\mathrm{VJ}$, hay evidencias en diversas investigaciones que permiten descartar la neutralidad o inocuidad de los juegos electrónicos, tal y como parece defender el psicólogo Estallo, y afirmar en algunos aspectos su influencia nociva, así como sus posibilidades favorables en el campo de la reeducación, determinados aprendizajes y la terapia.

A pesar de las limitaciones que siempre existen en el conjunto de la investigación, hemos hecho una revisión de 262 informes de investigaciones internacionales, realizados durante 1984 a 1996 [4] , y relacionados con los videojuegos. Quizás esto nos permita aportar un poco más de claridad sobre el extenso campo de los VJ

Los principales temas sobre los que se han centrado las numerosas investigaciones realizadas hasta el momento, especialmente a partir de los 90, son los siguientes:

1. Adicción,

2. Autoestima, 


\section{Aprendizaje,}

4. Cambios fisiológicos,

5. Entrenamiento,

6. Efectos negativos,

7. Espacial (habilidad),

8. Resolución de problemas

9. Sexo

10. Sociabilidad

11. Terapia

12. Violencia.

Muchos de estos informes sobre las investigaciones llevadas a cabo se han originado en Estados Unidos, y en aproximadamente un $50 \%$ de los casos no existen conclusiones sobre los resultados.

La literatura en lengua castellana es más reducida y la mayoría de los títulos no hacen referencia a investigaciones. Es por ello por lo que merece la pena destacar el trabajo de Juan Alberto Estallo (1995), y de Begoña Gros (1998), como unos de los pocos en los que se realiza una labor de investigación, además de un análisis a fondo de lo que suponen los videojuegos en el mundo actual. En el caso del primer autor es bastante incomprensible el "olvido" de una serie de estudios que concluyen de manera negativa sobre determinados aspectos de la personalidad de los jugadores y el balance tan optimista que hace del uso de los VJ.

\section{7.-Consecuencias de los VJ en algunos aspectos de la educación.}

\section{a).-Violencia y agresividad}

Los temas violentos son muy frecuentes en los VJ, sean en forma fantástica con monstruos, extraterrestres, alienígenas y otros tipos de animales, o en forma humana, basados en guerra, peleas callejeras y otros tipos de combates. A pesar de que la literatura sobre este asunto está suficientemente comprobada, que un repaso a las revistas especializadas sobre videojuegos ponen en evidencia y que prácticamente no admite discusión, nos hemos permitido ilustrar con una serie de juegos y sus características la primera afirmación: la presencia de la violencia en los videojuegos del mercado. Hemos analizado el contenido de la revista Hobby Consolas, $\mathrm{n}^{\circ}$ 82, 1998) y del conjunto de juegos que vienen presentados los hemos clasificado en dos categorías: violentos, sea de tipo humano, extraterreste, monstruos, etc. y no violentos, como los de Disney, fútbol, y otros.

Creemos interesante, para comprender el grado de violencia que ofrecen estos juegos, reproducir parte de los textos que acompañan a la presentación de los VJ. Los comentarios son muy elocuentes y no necesitan mayores ampliaciones: 


\begin{tabular}{|c|c|}
\hline $\begin{array}{l}\text { NOMBRE DEL } \\
\text { VIDEOJUEGO }\end{array}$ & CARACTERÍSTICAS VIOLENTAS \\
\hline BLUE STINGER & $\begin{array}{l}\text { "Uno de los videojuegos más terroríficos de la } \\
\text { historia" }\end{array}$ \\
\hline S.C.A.R.S. & $\begin{array}{l}\text { "Pulveriza a tus oponentes gracias a las armas } \\
\text { ultrapoderosas que te encontrarás en los } \\
\text { circuitos" }\end{array}$ \\
\hline ODDWORLD & $\begin{array}{l}\text { "Además de los enemigos de la primera parte, } \\
\text {... habrá sligs voladores que arrojan bombas..." }\end{array}$ \\
\hline PSYGNOSIS & $\begin{array}{l}\text { "Nos propone una de las aventuras más tétricas } \\
\text { y siniestras... con cientos de trampas, caídas } \\
\text { mortales... violentos actos que serán remojados } \\
\text { con sangre..." }\end{array}$ \\
\hline APOCALIPSE & "El fin de la guerra está cerca..." \\
\hline JIM, 3D & $\begin{array}{l}\text { "Dispara, gusano. Un bicho de (13) armas } \\
\text { tomar" }\end{array}$ \\
\hline TENCHU & $\begin{array}{l}\text { "No escatimará en escenas sangrientas, por lo } \\
\text { que será un juego no apto para todos los } \\
\text { públicos." }\end{array}$ \\
\hline TOMB RAIDER III & $\begin{array}{l}\text { "Encontraremos un mayor número de armas, } \\
\text { como un destructivo lanzacohetes..." }\end{array}$ \\
\hline XG2 & $\begin{array}{l}\text { "Aquí el objetivo pasa de ser el más rápido a } \\
\text { tener la mejor puntería, tratando de destruir a tus } \\
\text { "amigos" lo antes posible." }\end{array}$ \\
\hline MEDIEVIL & $\begin{array}{l}\text { "El juego en el que antes de empezar ya estás } \\
\text { muerto" }\end{array}$ \\
\hline TUROK & "La bestia despertará en noviembre" \\
\hline DEEP FEAR & $\begin{array}{l}\text { "El irresistible y profundo encanto del miedo". } \\
\text { Su defensa se basa en la lucha, aunque también } \\
\text { puede provocar explosiones de energía o lanzar } \\
\text { granadas". }\end{array}$ \\
\hline ASSAULT & $\begin{array}{l}\text { "...demostrando la increíble potencia que } \\
\text { pueden tener nuestras armas". }\end{array}$ \\
\hline BREATH OF FIRE & $\begin{array}{l}\text { "... vamos encontrándonos con enemigos que } \\
\text { nos atacan cada dos manosegundos... matamos a } \\
\text { los enemigos con asombroso desparpajo para el } \\
\text { espectador..." }\end{array}$ \\
\hline $\begin{array}{l}\text { DUKE NUKEM, } \\
\text { TIME TO KILL }\end{array}$ & "Es un juego en el que no dejarás de disparar". \\
\hline FUTURE COP & $\begin{array}{l}\text { "Acción a raudales, disparos ininterrumpidos, } \\
\text { explosiones constantes..." }\end{array}$ \\
\hline \begin{tabular}{|l} 
SHADOW OF \\
DARKNESS \\
\end{tabular} & $\begin{array}{l}\text { "Juego de trepidante acción en el que un ninja } \\
\text { debe enfrentarse a tropecientos enemigos" }\end{array}$ \\
\hline SMALL SOLDIERS & $\begin{array}{l}\text { "Nuestro objetivo consistirá en eliminar a todos } \\
\text { los soldaditos que podamos..." }\end{array}$ \\
\hline THRILL KILL & $\begin{array}{l}\text { "Médicos locos, caníbales, paranoicos } \\
\text { asesinos... Humor negro no apto para menores" }\end{array}$ \\
\hline
\end{tabular}




\begin{tabular}{||l||l||} 
TEKKEN 3 & $\begin{array}{l}\text { “El objetivo es aguantar en pié el mayor tiempo } \\
\text { posible y tumbar rivales a toda velocidad" }\end{array}$ \\
\hline \hline MORTAL KOMBAT & $\begin{array}{l}\text { "Es, de lejos, el más espectacular de la serie, el } \\
\text { más fuerte y el más agresivo. Salsa de tomate a } \\
\text { raudales...” }\end{array}$ \\
\hline \hline FORSAKEN & $\begin{array}{l}\text { “Es un juego impactante, demoledor... Lo ideal } \\
\text { es armarse con los misiles más potentes y } \\
\text { procurar moverse con rapidez". }\end{array}$ \\
\hline RESIDENT EVIL 2 & "Miedo?. (Tú no sabes aún lo que es el miedo)”. \\
\hline MACHINE HUNTER & "Preparado para disparar". \\
\hline \hline
\end{tabular}

FUENTE: Hobby Consolas, 1998

El tema de la violencia en los V.J. es uno de los principales tópicos (tal y como hemos podido comprobar, muy justificado) en la literatura sobre el estudio de los efectos de los videojuegos en la conducta infantil. Desde el comienzo de la difusión de los VJ, en los años 70 , la preocupación de padres y educadores se ha orientado hacia los supuestos peligros que el empleo de estos juegos puedan provocar en la infancia. Se han cruzado acusaciones desde los dos bandos: desde los que alarman de los peligros del uso de los VJ hasta quienes afirman que la mayoría de las investigaciones carecen de fundamento. Sin embargo, de entre la literatura al respecto, cabe destacar las voces de alarma de la APA (American Psychological Association), para quien las investigaciones demuestran que existe una correlación positiva entre la práctica de los VJ violentos y la conducta agresiva posterior.

También va en esta línea el estudio realizado por Bernad Cesarone (1994) quien afirma que el $71 \%$ de los VJ están clasificados en las categorías más altas, 1 a 3, en cuanto al índice de violencia. Por otra parte, de los $47 \mathrm{VJ}$ preferidos, 40 de ellos tenían como tema la violencia. Asimismo, según dicha investigación, y contrariamente a lo que indicaban las primeras investigaciones, las más recientes apoyan la tesis de que existe relación entre los niños que juegan VJ violentos y sus posteriores conductas agresivas. De igual modo, afirma que 9 de 12 estudios analizados revelan un fuerte impacto de los juegos violentos, que tienen consecuencias perjudiciales. Finalmente, en este estudio se llama la atención sobre los efectos negativos respecto a la agresividad y a los roles sexistas, que sobre todo proporcionan una imagen pasiva de las mujeres.

A pesar de que muchas investigaciones no son definitivas, las recomendaciones relacionadas con los VJ hacen una llamada a la prudencia en su uso, ya que numerosos estudios (Klemm, B. y otros, 1995; Ballard, M. -Wiest, R., 1995; Shutte, N. y otros, 1988; Anderson, C. y otros, 1986; Braun, C. y otros 1986) ponen de manifiesto que existe una relación entre la práctica de los VJ violentos y la conducta agresiva y otros problemas relacionados con ella

\section{El efecto de las críticas}

Las críticas de todo tipo que se han vertido sobre los efectos de los VJ en la formación de los niños y adolescentes han surtido algún tipo de reacción en las compañías de juegos electrónicos. Algunas de ellas se han visto obligadas a incluir en los juegos una serie de advertencias sobre determinados peligros para quienes utilizan estos sistemas de juego. Tanto Nintendo como Sega avisan a los usuarios sobre los riesgos que existen para 
determinados sujetos, especialmente los propensos a los ataques de epilepsia. Sega advierte: "Un porcentaje muy pequeño de individuos podrá sufrir ataques de epilepsia al exponerse a ciertos patrones de luz o luces destellantes". También Nintendo hace una llamada de atención en esta línea. Sega además contraataca defendiendo la necesidad del juego en la formación infantil: "En Sega hemos desarrollado unas videoconsolas estudiadas para incentivar el aprendizaje, los reflejos, la intuición... y un espíritu de superación que responde a sus necesidades".

Sin embargo, los VJ calificados como peligrosos no han desaparecido, sino que han recibido mayor impulso. Revisando los textos utilizados en las revistas que promocionan $\mathrm{y}$ ofrecen los nuevos juegos podemos encontrar una justificación argumentada de la defensa de los métodos y la ideología violenta contenida en los VJ. A modo de ejemplo veamos algunas de las expresiones utilizadas:

"Lejos de reformarse los programadores por las críticas recibidas por la violencia que exhibía Mortal Kombat y suavizar los combates, lo que han hecho ha sido hacerlos mucho más sangrientos, mostrándonos formas de morir realmente crueles y sorprendentes".

"Si alguien se escandaliza por la sangre que salpica por todas partes mientras dura el combate, que no mire cuando éste finaliza. ...acaban con los miembros y las partes del pobre desgraciado repartidos por toda la pantalla. Esta fué una de las partes más escabrosas de 'Mortal Kombat' que, lejos de atenuarse, se ha mantenido y desarrollado" (Micromanía, $\mathrm{n}^{\circ} 2$, p. 59)

La empresa que comercializa este juego ha introducido una solución salomónica para hacer frente a las críticas sobre la violencia en sus juegos. Esta solución consiste en permitir al usuario que pueda jugar en la "versión violenta" o en la versión regida por el "código de honor."

"Por tanto, cada jugador es libre de elegir el modo de juego que prefiera. Nadie está obligado a participar en un modo $u$ otro, y, en último caso, tampoco está obligado a comprarse el juego." (Acclaim Entertainment Inc. 1993, p. 36)

Según este texto, se reconocen los posibles efectos negativos del videojuego de lucha a muerte, pero se traslada al usuario, generalmente niños y adolescentes, la decisión del formato de juego que prefiere: versión violenta o versión de honor. Pero el problema fundamental no se resuelve, porque las personas que tienen que tomar esa decisión son justamente las que más riesgo tienen de ser influenciadas por los efectos negativos de los VJ. Tampoco parece un argumento muy sólido el afirmar que, "en último caso, tampoco está obligado a comprarse el juego".

Como era de suponer, este tipo de juegos se va desarrollando en su propia lógica imparable y en AMortal Kombat 4" (1998), se advierte al usuario que el juego es "de lejos, el más espectacular de la serie, el más fuerte y el más agresivo. Te avisamos, por tanto, que, a pesar de que se puede desconectar el modo gore, estamos ante un juego sólo para adultos... y para adultos curtiditos. "Salsa de tomate" a raudales, fatalities que asustan ....". (Hobby Consolas, $\mathrm{n}^{\mathrm{o}} 81,1998$ ).

Es de prever que haya una reacción ante los efectos de los $\mathrm{VJ}$, al igual que ha ocurrido en el mundo de la televisión, en el que las asociaciones de consumidores, los responsables educativos, y hasta las propias cadenas de televisión han asumido, por lo menos en el papel, la responsabilidad que tienen para establecer una cierta protección a los niños y 
adolescentes ante el constante bombardeo de escenas violentas en la pantalla.[5]

\section{b).-Videojuegos y género}

Ya hemos visto que los varones y las mujeres manifiestan diferentes preferencias y grados de adicción a los VJ. Pero también hay que tener en cuenta que los VJ juegan un papel importante en el tratamiento diferenciado que le dan a los roles masculinos y femeninos. Una crítica que tiene su origen en los primeros años de aparición de los VJ es la que afirma que las mujeres tienen un papel más pasivo, apareciendo en muchas ocasiones como víctimas, cautivas o en papeles secundarios, cuando no son ignoradas.

Provenzo (1992) halló, tras analizar las cubiertas de 47 de los juegos preferidos de Nintendo, que figuraban 115 varones y 9 mujeres. De estos varones, 20 tenían una actitud dominante, que no aparecía en ninguna mujer. 13 de las 47 cubiertas presentaban un escenario en el que las mujeres estaban apresadas o necesitaban ser rescatadas.

Numerosos estudios (Braun y otros, 1986; Strasburgber, V. y otros, 1993; Cesarone, 1994; Colwel y otros, 1995) revelan que la representación femenina en los videojuegos es menor, generalmente minusvalorada, y en actitudes dominadas y pasivas.

Solamente un número reducido de investigaciones manifiestan no hallar diferencias en el tratamiento a los chicos y las chicas, ni la existencia de estereotipias.

\section{Otras diferencias entre chicos y chicas con relación a los VJ}

Respecto al sexo de los jugadores, casi todos los estudios analizados afirman que los chicos tienen una mayor preferencia que las chicas por los $\mathrm{VJ}$, que dedican más horas y más frecuentemente y que los chicos tienen una mayor preferencia por los juegos agresivos.

En un análisis de las cartas de los participantes enviadas a 4 revistas especializadas en videojuegos, tanto para los concursos y los sorteos, así como para las consultas que realizan los usuarios, hemos comprobado que el numero de chicos supone un total de 273 $(92,5 \%)$ de las 283 referencias, mientras que las chicas constituyen únicamente 10 cartas (un $3,5 \%$ ). Este dato nos indica que una mayoría casi unánime de los usuarios representados son varones, mientras que las chicas significan una reducida proporción. Se confirma la información tantas veces ofrecida en el sentido de que los chicos juegan en mayor proporción que las chicas. Lo que no habíamos constatado era una proporción tan diferenciada entre varones y mujeres. Este dato puede indicar que los VJ, seguramente por su carácter violento y sexista, apenas despiertan el interés de las chicas.

En cuanto al rendimiento o nivel de resultados, también son bastante contundentes los estudios (Hall, E. 1990; Subrahmanyam, K. y otros, 1994; Kuhlman, J. y Beitel, P. 1991) que demuestran que las chicas y los chicos apenas difieren en cuanto a los resultados derivados del entrenamiento o aprendizajes a través del VJ. Tanto la autoconfianza, el desarrollo de las habilidades espaciales, así como otros tipos de aprendizajes no difieren con respecto al sexo de los jugadores.

\section{Tamagotchi, o el juego de mamás para niñas.}

Es el nombre que recibe un videojuego portátil que consiste en una mascota virtual que hay que atender como si se tratara de un bebé. Creado por Aki Maita para la casa Bandai, 
tras haber analizado el mercado de los juguetes, haber realizado cuestionarios y entrevistas y haber examinado las lecturas y aficiones de las niñas. El juego consiste en cuidar y criar de una mascota. Desde el momento en que despertamos al Tamagotchi "hay que alimentarle, limpiarle, curarle, jugar con él y hasta regañarle cuando no se porte bien.”. Al igual que una mascota real, la virtual irá creciendo y de la calidad de nuestros cuidados dependerá que el Tamagotchi se convierta en una linda mascota cibernética o en un alienígena mal educado y feo, así como que desee estar más o menos tiempo a nuestro lado. Concretamente, las funciones que el jugador debe realizar son las de atender a la comida, la luz, el juego, la inyección, el aseo, la salud, la educación y la atención. Tiene una vida de 28 días, equivalente a 28 años en su planeta virtual.

No hace falta ser muy astuto para concluir que este tipo de juego es la variante virtual del clásico "jugar a mamás" de nuestras niñas de antes y de ahora. Probablemente, esa es la razón por la que se explica el poco interés que despierta en los chicos, en general.

\section{c).-La inteligencia y los videojuegos}

Si bien las investigaciones no son definitivas, la mayoría de ellas indican que muchos videojuegos favorecen el desarrollo de determinadas habilidades, de atención, concentración espacial, resolución de problemas, creatividad, etc. por lo que se concluye que en su conjunto, desde el punto de vista cognitivo, los $\mathrm{VJ}$ suponen algún tipo de ayuda en el desarrollo intelectual (Mandinacht, E. 1987; White, B., 1984; Okagaki, L y Frensch, $\mathrm{P}, 1994)$. Se sugiere que quienes juegan a los VJ adquieren mejores estrategias de conocimiento, modos de resolver problemas, se benefician en sus habilidades espaciales y aumenta su precisión y capacidad de reacción. No hay evidencia de los efectos contrarios.

\section{d).-Sociabilidad y videojuegos}

Este es un tema que se ha estudiado también en muchas investigaciones, y que tiene un eco especial en las preocupaciones de padres y educadores, temerosos de que el apego de los niños y adolescentes hacia el $\mathrm{VJ}$ provoque un mayor aislamiento y reducción de contactos con sujetos de la misma edad.

Pues bien, la mayoría de las investigaciones que han analizado especialmente este aspecto de la personalidad de los jugadores han encontrado que los VJ, lejos de suponer un obstáculo para la práctica de las relaciones sociales, parecen estar relacionados con una mayor extroversión, una mayor frecuencia de trato con los amigos y una mayor socialización (Estallo, 1994; Been, C y Haring, Th., 1991; Shimai, Masuda y Kishimoto, 1990; Colwel, 1995; Fileni, F. 1988). Aunque no se pueda concluir una causalidad, "los VJ fomentan la sociabilidad", sí se puede afirmar que existe una fuerte relación: aquellos que son más jugadores tienen una mayor vida social, ven más a sus amigos, demuestran mayor extraversión y mayor iniciación social.

Es importante también destacar dos aspectos, tal y como ha quedado reflejado más arriba. Muchos de los juegos admiten más de un jugador y fomentan en cierto modo el juego en grupo. Por otra parte, aproximadamente un $70 \%$ de los jugadores de VJ afirman jugar acompañados, por lo que los supuestos efectos nocivos del juego solitario no parecen tener mucho fundamento. 
Del conjunto de investigaciones analizadas podemos sacar la conclusión de que el uso de los videojuegos en la ayuda para determinados aprendizajes y entrenamientos es muy positivo, tal y como se demuestra en el terreno del tratamiento de los problemas de aprendizaje, la ayuda para resolver problemas, para responder a cuestiones relacionadas con la escuela, las drogas, la familia, aspectos morales, etc. Los videojuegos permiten aumentar la motivación para el aprendizaje de diversas materias como las matemáticas y las ciencias, y el conjunto de las enseñanzas.

Además pueden ser utilizados como entrenamiento eficaz en programas de tipo visomotor, desarrollo del pensamiento reflexivo, mejora de las habilidades de los pilotos de avión, reducir el número de errores de razonamiento, predictores de los tests de lápiz y papel, mejorar la eficacia de los trabajadores sociales, conseguir un mayor control de los tiempos de reacción, y servir de enfrentamiento ante situaciones vitales que pueden ser simuladas, como es el caso de la resolución de problemas, tema en el que se muestran muy eficaces.

\section{9.-El uso terapeútico de los VJ}

Si bien el uso educativo de los VJ no está muy extendido, o por lo menos no existen muchas referencias bibliográficas sobre investigaciones al respecto, en el tema de la reeducación, las dificultades de aprendizaje, la terapia psicológica y fisiológica cuenta con abundantes trabajos que se han llevado a cabo utilizando los videojuegos.

Entre las investigaciones que se han realizado y de las que contamos con algún tipo de conclusiones (aproximadamente unas 30), podemos afirmar que, en la mayoría de los casos, los resultados obtenidos han sido satisfactorios, reforzando la idea ya anteriormente sostenida de que la utilización de esta nueva tecnología produce mejora en el rendimiento, la reeducación o la recuperación de algunas destrezas o habilidades de tipo físico o psicológico.

Las principales áreas analizadas han sido las relacionadas con los siguientes aspectos de la personalidad:

- Habilidades de relación y comunicación entre niños

- Trastornos del lenguaje

- Desarrollo de la coordinación visomotriz

- Mejora de sujetos con múltiples handicaps

- Reducción de conductas antisociales

- Conductas impulsivas

- Aumento del autocontrol en jóvenes delincuentes

- Reducción de conductas autodestructivas

- Desarrollo de la cooperación

- Reducción de la ansiedad

- Toma de decisiones respecto a las drogas

- Regulación de la tensión arterial y presión sanguínea 
Como muestra del optimismo respecto al uso de los VJ en el tratamiento de determinados problemas, citaremos el trabajo de Casey, J.A (1992) relativo al uso de tecnología en la tutoría con jóvenes en riesgo. Las conclusiones de este estudio defienden que existe una serie de ventajas, tal y como se había detectado en un principio: los jóvenes tienen generalmente una buena relación con el uso de los VJ; el aprendizaje encubierto puede sustituir al aprendizaje formal, venciendo la normal resistencia. Además, la representación multisensorial del aprendizaje, utilizando imágenes, sonido y modalidades kinestésicas facilita más la enseñanza. Por otra parte, el aprendizaje individual puede permitir el logro de objetivos más realistas, superando las dificultades del miedo al público o al grupo.

Para Gifford (1991), existen siete características que hacen de los VJ un medio de aprendizaje más atractivo y efectivo:

1. Permiten el ejercicio de la fantasía, sin limitaciones espaciales, temporales o de gravedad.

2. Facilitan el acceso a "otros mundos" y el intercambio de unos a otros a través de los gráficos, contrastando de manera evidente con las aulas convencionales y estáticas.

3. Favorecen la repetición instantánea y el intentarlo otra vez, en un ambiente sin peligro.

4. Permiten el dominio de habilidades. Aunque sea difícil, los niños pueden repetir las acciones, hasta llegar a dominarlas, adquiriendo sensación de control.

5. Facilitan la interacción con otros amigos, además de una manera no jerárquica, al contrario de lo que ocurre en el aula.

6. Hay una claridad de objetivos. Habitualmente, el niño no sabe qué es lo que está estudiando en matemáticas, ciencias o sociales, pero cuando juega al VJ sabe que hay una tarea clara y concreta: abrir una puerta, rescatar a alguien, hallar un tesoro, etc. lo cual proporciona un alto nivel de motivación.

7. Favorece un aumento de la atención y del autocontrol, apoyando la noción de que cambiando el entorno, no el niño, se puede favorecer el éxito individual.

Como ya hemos dicho, la mayoría de las investigaciones realizadas tienen un balance positivo para el uso de los VJ en la práctica de las diversas terapias. Tanto los niños como los autores se muestran satisfechos y la mejora en las diferentes facetas tratadas parece ser evidente. Por todo ello podemos concluir que los VJ son un instrumento adecuado para conseguir mejorar o reeducar determinados aspectos de las personas, en mayor medida que el uso de los métodos convencionales.

\section{0.-Conclusiones.}

Después de lo mucho que se ha escrito e investigado sobre los VJ, podemos concluir que es un tema que está adquiriendo una importancia creciente como objeto de estudio por parte de educadores, psicólogos, sociólogos y médicos, principalmente.

En el marco concreto de la educación podemos distinguir diversos efectos de los videojuegos. Hay algunos rasgos que tienen carácter negativo, y hay otros en los que incluso se puede detectar influencias positivas o usos constructivos y beneficiosos. 


\section{A.-Los peligros de los videojuegos actuales en el mercado: violencia y sexismo}

Según las investigaciones que hemos podido analizar, los dos aspectos incontestables en cuanto al balance negativo de la influencia de los videojuegos en la personalidad de los jugadores son los efectos perjudiciales en el terreno de la violencia y del sexismo. La mayoría de los VJ fomentan las actitudes violentas y agresivas, que como se ha comprobado tienden a repetirse en la conducta de los niños y adolescentes.

Por otra parte, es también evidente la existencia de estereotipos en cuanto a las figuras masculinas y femeninas que van en perjuicio de las mujeres, puesto que aparecen en menor proporción, y cuando lo hacen tienden a ser representadas en actitudes pasivas, dominadas o secundarias, mientras que los varones están más representados, en actitudes más activas y dominadoras.

\section{B.-Los aspectos positivos de los videojuegos: la sociabilidad, la inteligencia y el uso educativo y terapéutico.}

A pesar de las críticas recibidas también en este terreno, los videojuegos no se muestran como desencadenantes de un deterioro de las relaciones sociales de los jugadores. Por el contrario, la afición a los VJ está relacionada con actitudes positivas de socialización.

Por otra parte, la inteligencia no parece sufrir ningún tipo de deterioro por la utilización de los VJ. Por el contrario, se concluye que el juego con VJ favorece el desarrollo de determinados aspectos de la inteligencia, sobre todo los de carácter espacial.

Finalmente, se ha demostrado de manera contundente que los videojuegos permiten una ayuda especial en el tratamiento y mejora de problemas educativos y terapéuticos, tanto de tipo físico como psicológico, así como múltiples utilidades en cuanto al entrenamiento de todo tipo de habilidades.

\section{C.-No son causantes de patologías especiales.}

Parece que los VJ pueden desencadenar problemas en un número reducido de sujetos que presentan una predisposición anterior, como la epilepsia, y por otra parte se ha demostrado que tienen un influjo estresante y favorecedor de la ansiedad, con alteración de la presión sanguínea y el ritmo cardiovascular. Sin embargo, los supuestos trastornos psicopatológicos derivados del juego electrónico no parecen estar confirmados en las investigaciones realizadas hasta ahora.

\section{1.-Bibliografía.}

Anderson, C. y otros, (1986): "Affect of the game player: short term effects of highly and midly aggressive video games." Personality and Social Psychology Bulletin. Vol 12(4) 390-402. Houston.

Ballard, M. - Wiest, R. (1995): "Mortal kombat: the effects of violent video technology on males' hostitily and cardiovascular responding." Biennal Meeting of the Society for Research in Child Development. March 30 april 2. Indianapolis.

Bandura, A. (1984): Teoría del aprendizaje social. Espasa-Calpe. Madrid.

Barrueco, V. (1993): "Videojuegos: la fiebre de fin de siglo". IP. MARK. (405): 38-40, 9 REF. Universidad Autónoma de Madrid 
Been, C y Haring, Th. (1991): "Effects on contextual competence on social initiations". Journal of Applied Behavior Analysis. Sum vol 24(2) 337-347. California.

Braun, C. y otros (1986): "Adolescents and microcomputers: Sex differences, proxemics, task and stimulus variables." Journal of Psychology. Nov Vol 120(6) 529-542. Montreal. Canada

Casey, J. \& Ramsammy, R. (1992): "MacMentoring: Using techonology and counseling with at-risk youth.” ERIC Document Reproduction Service. No, ED 344179.

Castillejo, J.L. (1987): Pedagogía Tecnológica. CEAC. Barcelona.

Cesarone, B. (1994): "Videogames and children". Office of Educational Research and Improvement. Washington

Clark, C.S. (1993): “TV violence.” "CQ Researcher" 3 (12, Mar 26) 167-187

Colwell, J. y otros (1995): "Computer games, self esteem and gratification of needs in adolescents". Journal of Community and applied social psychologi. 5(3): 195-206

Estallo, J.A. (1994): “Videojuegos, personalidad y conducta". Psicothema. 6(2) 181-190, $16 \mathrm{REF}$

Estallo, J.A. (1995): Los videojuegos: juicios y prejuicios. Planeta. Barcelona.

Etxeberria, F. (1999): "Videojuegos y educación", en Etxeberria, F. (Coord): La Educación en Telépolis. Editorial Ibaeta. Donostia.

Fernandez, L. y Marin, I. (1992): "Los videojuegos enganchan". Perspectiva escolar. (169) 57-63

Fileni, F. (1988): "Play as acquisition of mental structures: the case of videogames." Studi di sociologia. Jan-mar, 64-74. Calabria.Italia.

Funk, J.B. (1993): "Reevaluating the impact of Video Games." Clinical Pediatrics 32 (2, Feb): 86-90. PS 521 243)

Gaja, R. (1993): Videojuegos ¿Alienación o desarrollo?. Editorial Grijalbo. Barcelona.

Gifford, B.R. (1991): "The learning society: Serious play". Chronicle of Higher Education, p. 7

Gros, B. (Coord) (1998): Jugando con videojuegos: Educación y entretenimiento. Editorial Desclee de Brouwer. Bilbao.

Hall, E. (1990): "The effect of perfomer gender, perfomer skill level, and opponent gender on self-confidence in a competitive situation". Journal of Research; v23 n1-2 p33 jul.

Henderson, H.J. (1989): Counseling with computers: Technology and techniques. Lancaster, TX: 3S Co.

Kinder, M. (1991): Playing with power in Movies, Television and Video Games: From Muppet Babies to teenage Mutant Ninja Turtles. University of California Prees. Berkeley. 
Klemm, B. and others (1995): "Various viewpoints on violence". Young-children; v50 n5 p53-63 jul

Kubey, R. and Larson, R. (1990): “The use and Experience of the New Video Media among children and Young Adolescents". Communication Research. 17(1): 107-130. EJ 406646

Kuhlman, J. and Beitel, P. (1991): "Videogame experience: a possible explanation for differences in anticipation of coincidence". Perceptual and motor skills. Apr vol 72(2) 483-488. Indiana.

Levis, D. (1997): Loa videojuegos, un fenómeno de masas. Editorial Paidós. Barcelona.

López, E. y Ortega, J.M. (1982): El juego: entre la habilidad y el azar. Aula abierta Salvat. Barcelona.

Mandinacht, E. (1987): "Clarifying the "A" in CAI for learners of different abilities." Journal of Educational Computing Research. vol 3(1) 113-128. Princeton. USA

Margolies, R. (1991): “The computers as a social skills agent.” T.H.E. Journal, 18(6), 7071

National Coalition on Television Violence. (1990): "Nintendo Tainted by Extreme Violence". "NCTV News" 11(1-2, Feb-Mar): 1, 3-4

Okagaki, L y Frensch, P. (1994): "Effects of video game playing on measures of spatial perfomance: gender effects in late adolescence". Journal of Applied Development Psychology. Jan-Mar vol 15(1) 33-58

Perez, M.A. y Lopez, J. (1993) "Los videojuegos como nueva realidad social y cultural”. Infancia y Sociedad. N1 20.

Perez, P.M. (1994): Los niños y los valores. Instituto de Creatividad e Innovaciones educativas de la Universidad de Valencia. S.M. Valencia.

Provenzo, E. (1991): Video kids: Making sense of Nintendo. Harward University Press. Cambrigde

Provenzo, E. (1992): “The Video Generation”. American School Board Journal. 179(3, Mar): 29-32. EJ 441136

Shimai, Masuda y Kishimoto, (1990): "Influences of TV games on physical and psychological development of Japanese kindergarten children". Perceptual and motor skills. Jun vol 70(3, pt 1) 771-776. Japan.

Shutte, N. y otros, 1988; "Effects of playing videogames on children's aggressive and other behaviors". Journal of Applied Social Psychology. Apr vol 18(5) 454-460

Strasburgber, V. y otros (1993): "Adolescents and the media." Adolescents-Medecine: State of the Art Reviews. V4 n3 oct. Philadelphia.

Subrahmanyam, K. y otros (1994): "Effect of video game practice on spatial skills in girls and boys". Journal of applied Developmental Psychology. Jan-Mar vol 15(1) 13-32. Los 
Angeles.

Turkle, S. (1986): Les enfants de l'ordinateur. Denöel. Paris.

White, B., (1984): "Designing computer games to help physics students understanding Newton's laes of motion". Cognition and Instruction. vol 1(1) 69-108. Cambrigde. USA

\section{NOTAS}

[1] Esta breve historia de los videojuegos puede ser ampliada con la obra del psicólogo Juan Alberto Estallo (1995): Los videojuegos. Juicios y prejuicios. Planeta. Barcelona.

[2] Así se constata en las investigaciones llevadas a cabo en la Universidad de Valencia, dirigidas por la profesora P.M. Perez Alonso-Geta. En concreto, en el año 1992, se analizaron 1.600 niños de toda España, con edades comprendidas entre 9 y 14 años.

[3] A modo de ejemplo, reseñamos la revista "Escuela de Salud", $n^{\circ}$ 12, del mes marzoabril de 1993, en su página 12, en el artículo "Videojuegos con riesgo" afirma: "Según los expertos, si se utilizan diaria y continuamente se facilita el en niño la aparición de numerosos trastornos físicos y psicológicos, que pueden llegar a alterar su conducta y a transformarse en graves problemas psicopatológicos".

[4] Hemos consultado el sistema ERIC, PsycLIT, y una selección de bases de datos, de libros, artículos de revistas, tesis doctorales, etc. facilitados por el banco de datos de la Universidad del País Vasco. El número de investigaciones que hemos revisado ha sido de 262.

[5] En 1992, la Asociación de Telespectadores y Radioyentes afirmaba que un niño europeo en edad escolar ve en una semana 67 homicidios, 15 secuestros, 848 peleas, 420 tiroteos, 30 casos de torturas, 18 de drogas y otra serie de emisiones eróticas.

En 1993, las televisiones privadas y públicas se comprometieron a firmar un convenio con las autoridades educativas estatales con el fin de establecer un marco de conducta para proteger a la infancia y la juventud.

(C) Ediciones Universidad de Salamanca. 